\title{
Deciphering the microRNA transcriptome of skeletal muscle during porcine development
}

Miaomiao Mai, Long Jin, Shilin Tian, Rui Liu, Wenyao Huang, Qianzi Tang, Jideng Ma, An'an Jiang, Xun Wang, Yaodong Hu, Dawei Wang, Zhi Jiang, Mingzhou Li, Chaowei Zhou, Xuewei Li

MicroRNAs (miRNAs) play critical roles in many important biological processes, such as growth and development in mammals. Various studies of porcine muscle development have mainly focused on identifying miRNAs that are important for fetal and adult muscle development; however, little is known about the role of miRNAs in middle-aged muscle development. Here, we present a comprehensive investigation of miRNA transcriptomes across five porcine muscle development stages, including one prenatal and four postnatal stages. We identified 404 known porcine miRNAs, 118 novel miRNAs, and 101 miRNAs that are conserved in other mammals. A set of universally abundant miRNAs was found across the distinct muscle development stages. This set of miRNAs may play important housekeeping roles that are involved in myogenesis. A short time-series expression miner analysis indicated significant variations in miRNA expression across distinct muscle development stages. We also found enhanced differentiation- and morphogenesis-related miRNA levels in the embryonic stage; conversely, apoptosis-related miRNA levels increased relatively later in muscle development. These results provide integral insight into miRNA function throughout pig muscle development stages. Our findings will promote further development of the pig as a model organism for human age-related muscle disease research. 


\section{Deciphering the microRNA Transcriptome of Skeletal 2 Muscle during Porcine Development}

3 Miaomiao Mai ${ }^{1, \dagger}$, Long Jin ${ }^{1, \dagger}$, Shilin Tian ${ }^{1,2}$, Rui Liu ${ }^{1}$, Wenyao Huang1, Qianzi Tang1, Jideng

$4 \mathrm{Ma}^{1}$, Anan Jiang1, Xun Wang1, Yaodong Hu${ }^{1}$, Dawei Wang², Zhi Jiang², Mingzhou Li', 5 Chaowei $\mathrm{Zhou}^{3, *}$ and Xuewei $\mathrm{Li}^{1, *}$

61 College of Animal Science and Technology, Sichuan Agricultural University, Ya'an, People's

7 Republic of China.

82 Novogene Bioinformatics Institute, Beijing, People's Republic of China.

93 Department of Aquaculture, Southwest University at Rongchang, Chongqing, People's

10 Republic of China.

$11 \dagger$ These authors contributed equally to this work.

$12 *$ Corresponding author:

13 Xuewei Li, xuewei.li@sicau.edu.cn; Chaowei Zhou, zcwlzq666@163.com

Abstract: MicroRNAs (miRNAs) play critical roles in many important biological processes, such as growth and development in mammals. Various studies of porcine muscle development

17 have mainly focused on identifying miRNAs that are important for fetal and adult muscle development; however, little is known about the role of miRNAs in middle-aged muscle

19 development. Here, we present a comprehensive investigation of miRNA transcriptomes across

20 five porcine muscle development stages, including one prenatal and four postnatal stages. We

21 identified 404 known porcine miRNAs, 118 novel miRNAs, and 101 miRNAs that are conserved

22 in other mammals. A set of universally abundant miRNAs was found across the distinct muscle

23 development stages. This set of miRNAs may play important housekeeping roles that are involved

24 in myogenesis. A short time-series expression miner analysis indicated significant variations in 25 miRNA expression across distinct muscle development stages. We also found enhanced 
26 differentiation- and morphogenesis-related miRNA levels in the embryonic stage; conversely, 27 apoptosis-related miRNA levels increased relatively later in muscle development. These results 28 provide integral insight into miRNA function throughout pig muscle development stages. Our 29 findings will promote further development of the pig as a model organism for human age-related 30 muscle disease research.

\section{Introduction}

Skeletal muscle makes up approximately $40 \%$ of mammalian body mass (Güller \& Russell 2010), and maintaining skeletal muscle function is a prerequisite for maintaining normal body function. Therefore, understanding how skeletal muscle grows is important for understanding the growth of the body as a whole. Numerous conditions, including neuromuscular disorders, sedentary lifestyles, chronic disease, and aging, have been shown to be associated with loss of skeletal muscle mass and function (Di Giovanni et al. 2004; Dogra et al. 2007; Jagoe \& Goldberg 2001; Lynch 2001). The growth of skeletal muscle mass, like the mass of any other tissue, depends on protein turnover (synthesis, degradation, and repair capacities) and cell turnover (differentiation and proliferation) (Sartorelli \& Fulco 2004). Cellular turnover plays a major role in embryonic muscle development. Moreover, postnatal muscle development is mainly associated with accumulation of myonuclei (satellite cell proliferation) and muscle-specific proteins (Rehfeldt et al. 2000). Conversely, the decrease in muscle mass, strength, and rate of contraction is closely related to aging, and these age-related changes have been observed in many species, including humans (Evans \& Lexell 1995), rats (Daw et al. 1988; Hooper 1981), and mice (Brooks \& Faulkner 1988). Notably, it is well documented that the remarkable structural and functional changes in skeletal muscle that occur during aging, including reduction in muscle mass and increased apoptosis (Dirks \& Leeuwenburgh 2002; Janssen et al. 2000; Jemal et al. 2008; Nair 2005; Navarro 
51 MicroRNAs (miRNAs) are endogenous small non-coding RNAs that modulate gene expression 52 at a post-transcriptional level by binding to the $3^{\prime}$ untranslated region ( $3^{\prime}$-UTR) of target mRNAs 53 (Nelson et al. 2003). To date, numerous miRNAs have been shown to be associated with skeletal 54 muscle aging and act as regulators of cellular senescence at the tissue or organism level. For example, Hamrick et al. (Hamrick et al. 2010) reported that miR-7, miR-468, miR-542, and miR698 levels in mouse muscle tissue substantially increased with age, whereas miR-124a, miR-181a, miR-221, miR-382, miR-434, and miR-455 levels substantially decreased with age. Similar findings have also been found in humans, in which 18 miRNAs were differentially expressed between younger and older men (Drummond et al. 2011). However, these findings are not sufficient to comprehensively understand the relationship between miRNAs and muscle 61 development, and further research is required.

The pig (Sus scrofa) harbors tremendous biomedical importance as a model organism, because of its closer phylogenic relationship and more similar physiology to humans than mice or other mammals (Lunney 2007). Although studies have identified changes in miRNA expression during muscle development in pigs (Chen et al. 2012; Hou et al. 2012; Huang et al. 2008b; Li et al. 2012; Xie et al. 2011; Zhou et al. 2013), only a small subset of ages was analyzed. Moreover, these studies mainly focused on understanding miRNAs involved in fetal and adult muscle development. Until now, little was known about the role of miRNAs in middle-aged muscle development.

Here, to identify miRNAs and determine their role in skeletal muscle development, we evaluated miRNA transcriptome profiles at five stages of muscle development, including one prenatal stage (90-d post-gestation fetuses, E90) and four postnatal stages ( $0 \mathrm{~d}, 30 \mathrm{~d}, 180 \mathrm{~d}$, and $7 \mathrm{y}$ after birth), which represented prenatal, natal, weaning, young adult, and middle-aged stages, respectively (Bhathena et al. 1996). Our study will contribute to understanding the underlying mechanisms of muscle development and muscular atrophy.

\section{Methods}




\section{Animal ethics statement}

7 All animals used in this study were farmed according to the Regulations for the Administration of Affairs Concerning Experimental Animals (Ministry of Science and Technology, China, revised in June 2004) and approved by the Institutional Animal Care and Use Committee in the College of Animal Science and Technology, Sichuan Agricultural University, Sichuan, China under permit No. DKY-B20121406.

\section{Preparation of experimental animals and tissues}

3 Fifteen female Jinhua pigs, which were grouped into five stages (three replicates for each stage): prenatal embryonic day 90 (E90), postnatal $0 \mathrm{~d}$ (natal), $30 \mathrm{~d}$ (weaning), $180 \mathrm{~d}$ (young adult), and 7 y (middle-aged), were humanely sacrificed. The longissimus dorsi muscle tissues from the same anatomical location were collected from pigs representing each of the five stages. Subsequently, the samples were immediately frozen in liquid nitrogen and stored at $-80^{\circ} \mathrm{C}$ until RNA extraction. Total RNA was isolated from each sample using Trizol (Takara) according to the manufacturer's protocol. The concentration and purity of RNA samples were determined by measuring the A260/A280 ratio using a NanoDrop ND1000 spectrophotometer (Thermo Scientific, Hudson, NH, USA).

\section{Myofiber histology}

3 After sacrifice, all muscle tissues were fixed in 10\% neutral buffered formalin solution, embedded in paraffin using a TP1020 semi-enclosed tissue processor (Leica), sliced at a thickness of $6 \mu \mathrm{m}$ using RM2135 rotary microtome (Leica), and stained with hematoxylin and eosin (H\&E).

The mean diameter of muscle cells was calculated as the geometric average of the maximum and minimum diameter, and 100 cells were measured for each sample in randomly selected fields using a TE2000 fluorescence microscope (Nikon) and Image Pro-Plus 7.0 software. 
99 Small RNA library construction and high-throughput sequencing

100 We pooled the total RNA for replicates in the E90, 0-d, and 30-d stages. For 180-d and 7-y stages,

101 total RNA of each replicate was individually used for library construction. Therefore, a total of

102 nine libraries were constructed and sequenced using single-end sequencing in $36 \mathrm{nt}$ reads by an

103 Illumina Genome Analyzer II. The bioinformatics pipeline for miRNA discovery and profiling

104 was carried out as previously described, with some improvements (Gambardella et al. 2010). All

105 reads were counted and the identical reads were combined into a single kind. After eliminating

106 adaptor sequences, low-quality tags, sequences smaller than $16 \mathrm{bp}$, and reads with no insertion, all

107 of the clean tags were annotated and classified by comparison with the non-coding RNAs (rRNA,

108 tRNA, scRNA, snRNA, and snoRNA) in the GenBank (http://www.ncbi.nlm.nih.gov/) and Rfam

1099.1 (http://rfam.sanger.ac.uk/) databases. Known miRNAs were identified by comparing our clean

110 tags to mature miRNAs in miRBase 19.0. The remaining non-annotated sRNA sequences were

111 aligned against the porcine genome, and genomic sequences containing the sRNA with 80,100 nt

112 of flanking sequences were used to predict hairpin structures using Mfold

113 (http://mfold.rna.albany.edu). Only the sequences that could be folded into typical hairpin

114 structures and were located in intergenic regions or introns were considered to be miRNA

115 precursor loci of potential novel miRNAs in the porcine genome.

116 miRNA differential expression and clustering analyses

117 IDEG6 (Romualdi et al. 2003) was employed to detect DE miRNAs between two libraries. A

118 unique miRNA was considered DE when $\mathrm{P}<0.001$ was yielded by three statistical tests (Greller

119 and Tobin, R of Stekel and Falciani, and general chi-squared tests) with Bonferroni correction.

120 Hierarchical clustering analysis (HCL) of DE miRNAs was performed with MultiExperiment

121 Viewer (MeV) (Howe et al. 2010).

\section{Prediction and functional annotation of miRNA target genes}


123 The potential targets of a certain miRNA were predicted by PicTar(Krek et al. 2005)and

124 TargetScan Human 6.2 (Lewis et al. 2005), and the pairwise overlaps of the results from both

125 programs composed the final predicted targets. The predictions were based on human mRNA-

126 miRNA interactions, because of the absence of porcine miRNAs in the current version of

127 abovementioned algorithm. The enriched GO biological process (GO-BP) and Kyoto

128 Encyclopedia of Genes and Genomes (KEGG) pathway terms of predicted target genes were

129 determined using the DAVID bioinformatics resource (Huang et al. 2008a).

\section{STEM analysis}

131 STEM analysis was used to visualize expression patterns for DE miRNAs. Each miRNA was 132 assigned to the model profile that its time series most closely matched based on the correlation 133 coefficient. The number of miRNAs assigned to each model profile was then computed. The 134 number of miRNAs expected to be assigned to a profile was estimated by randomly permuting the 135 original time point values, renormalizing the miRNA expression values, assigning miRNAs to 136 their most closely matching model profiles, and repeating this process for a large number of 137 permutations. The average number of miRNAs assigned to a model profile over all permutations 138 was used as the estimate of the expected number of miRNAs assigned to the profile. The statistical 139 significance of the number of miRNAs assigned to each profile compared with the expected 140 number was also computed (Ernst \& Bar-Joseph 2006).

\section{1 qRT-PCR validation}

142 The expression changes of nine selected miRNAs were validated using an EvaGreen-based 143 High-Specificity miRNA qRT-PCR Detection Kit (Stratagene, La Jolla, CA, USA) on the 144 CFX96 $^{\mathrm{TM}}$ Real-Time PCR Detection System (Bio-Rad, Hercules, CA, USA). The q-PCR 145 validations were carried out on three biological replicates. Information on the primer pairs used is 146 available in Table S1. Three endogenous control genes (U6 snRNA, 18S rRNA, and 5S rRNA) 
147 were used in this assay. The $\Delta \Delta \mathrm{Ct}$ method was used to determine the expression level differences

148 between surveyed samples.

149 Results and Discussion

150 Phenotypic measurements

151 In this study, we investigated muscle fiber diameter and body weight change during the postnatal

152 muscle development stage. As shown in Fig. S1A-C, we found that muscle fiber diameter and

153 body weight were significantly increased ( $\mathrm{P}<0.001$, one-way analysis of variance) from $0 \mathrm{~d}$ to 7

$154 \mathrm{y}$, which is consistent with results from previous studies in pigs (Dwyer et al. 1993; Fiedler et al.

155 1997). Notably, we found that muscle fiber diameter and body weight growth rate significantly

156 slowed with age $(\mathrm{P}<0.05)$ (Fig. 1A, B). This age-related decrease of muscle fiber growth rate is

157 likely due to decreases in protein synthesis and increases in protein degradation (Jackman \&

158 Kandarian 2004). These phenotypic differences indicated possible underlying intrinsic differences

159 in molecular mechanisms.

\section{Summary of deep-sequencing data}

161 To investigate miRNA expression changes during pig muscle development, nine small RNA

162 libraries were constructed using deep sequencing. In total, 85.84 million raw reads (with

163 redundancy) were obtained. More than 56.67\% reads (ranging from $56.67 \%$ to $81.25 \%$ ) in each

164 library passed the Adapter (ADT) dimmer \& length, junk, mRNA, RFam, and Repbase filters and

165 were considered high-quality reads (Fig. S2). Of these high-quality reads (Fig. S3), the majority $166(90.35 \pm 1.34 \%, \mathrm{n}=9)$ of the small RNAs were approximately $21-24 \mathrm{nt}$ in length. More than half 167 of the reads were $22 \mathrm{nt}$ in length $(57.03 \pm 7.53 \%, \mathrm{n}=9)$, followed by $23 \mathrm{nt}(16.40 \pm 4.34 \%, \mathrm{n}=$ $1689), 21 \mathrm{nt}(12.37 \pm 5.26 \%, \mathrm{n}=9)$, and $24 \mathrm{nt}(4.55 \pm 1.49 \%, \mathrm{n}=9)$, which are typical lengths of 169 Dicer-processed products (Bartel 2009; Berezikov 2011). This result indicates that small RNA 170 sequencing is a reliable approach to generate miRNA reads for further analysis. 
172 The high-quality reads (sequences) were compared against the pig genome and divided into

173 three groups (Table 1): porcine known miRNAs, porcine conserved miRNAs, and porcine putative 174 new miRNAs. Of the porcine known miRNAs, 404 corresponded to 233 porcine known premiRNAs in miRBase 19.0 (http://www.mirbase.org/) and could be mapped to the pig genome. Specifically, 286 miRNAs were known, but 118 were not previously identified porcine miRNAs (Table S2). Of the porcine conserved miRNAs, 101 corresponded to 71 other known mammalian species except for porcine pre-miRNAs in miRBase 19.0, which were further mapped to the pig genome (Table S3). Of the porcine putative new miRNAs, 431 corresponded to 325 candidate premiRNAs that were not mapped to mammalian pre-miRNAs in miRBase 19.0 , but predicted RNA hairpins derived from the pig genome, and the extended genome sequences form hairpins, which were labeled PN (Table S4). Therefore, 629 pre-miRNAs that encode 936 mature miRNAs were identified. Because there are distinct pre-miRNAs and genomic loci that code identical mature sequences, 903 unique miRNAs were finally identified (Table S5).

To uncover the possible roles of these abundant miRNAs in each stage, we ranked the miRNAs by expression level. The profile of miRNA expression abundance was different across different development stages. As shown in Fig. S4, the most abundant miRNAs (top 10 unique miRNAs) account for more than $50.58 \%$ counts of all 903 unique miRNAs. Particularly, the counts of top 10 miRNAs occupied about $90 \%$ of the total counts in the $180-\mathrm{d}$ and $7-y$ libraries, which is significantly higher than that of the E90, 0-d, and 30-d libraries. Furthermore, the set of the top 10 unique miRNAs over the five muscle development stages corresponded to 16 kinds of unique miRNAs (Fig. 2). Of these miRNAs, six (miR-133a-1/-2-3p, let-7a-1/-2-5p, miR-27b-3p, miR$26 a-5 p$, miR-1-3p, and let-7f-1/-2-5p) were shared by all five stages and were closely related to myogenesis, cell growth, myocyte proliferation, and cell apoptosis. For example, miR-133a is a muscle-enriched miRNA that inhibits proliferation of progenitor cells and promotes myogenesis 
196 by targeting SRF (McCarthy \& Esser 2007). miR-26a promotes myogenesis in C2C12 cells via

197 post-transcriptionally repressing Ezh2, which is a known suppressor of skeletal muscle cell 198 differentiation (Wong \& Tellam 2008). These two miRNAs are most abundant during the fast199 growing stage of pig muscle. Moreover, miR-27a/b, a potential regulator of myogenesis, could 200 induce skeletal muscle hypertrophy by down-regulating myostatin, an inhibitor of myogenesis 201 (Huang et al. 2012; Sharma et al. 2014) and miR-27b inhibition leads to more proliferation and 202 delays the onset of differentiation (Crist et al. 2009). miR-1, a muscle-specific microRNA, 203 promotes cell apoptosis by targeting Bcl-2 (Tang et al. 2009), and could target heat shock protein 20470 (HSP70) in the development of muscle atrophy (Kukreti et al. 2013). Therefore, miR-27b 205 promotes myogenesis and proliferation, whereas miR-1 inhibits these processes and induces 206 apoptosis. miR-27b showed increasing expression levels during development, and miR-1 showed 207 the opposite trend. These results may indicate fluctuation of miRNA regulation during muscle 208 development.

209 In addition, 10 other miRNAs (miR-378-1/-2-3p, miR-127-3p, miR-191-5p, miR-486-2-5p, 210 miR-143-3p, miR-10a-5p, miR-148a-3p, miR-99a-5p, miR-30e-5p, and miR-199a-1/-2-5p) (Fig.

2112 ) in the set of the top 10 most highly expressed unique miRNAs over the five muscle development 212 stages are related to various cell proliferation, myogenesis, and apoptosis responses. For example, 213 miR-378 promotes myogenesis in pigs through regulation of bone morphogenetic protein 2 214 (BMP2) and mitogen-activated protein kinase 1 (MAPK1), especially in fiber formation in both 215 the fetal and newborn periods (Hou et al. 2012). In our study, miR-378 showed the highest 216 expression level in the E90 stage. Though Hou et al. found that the expression level of miR-378 217 increased at 65 and $90 \mathrm{dpc}$ and peaked at postnatal day 0 , these results both suggested that miRNA218378 was a new candidate miRNA for myogenesis in pigs (Hou et al. 2012; Qin et al. 2013). These 219 results supports the idea that it has an important role in fetal muscle development. Another miRNA, 220 miR-148a, belong to the top ten expressed miRNAs only in E90 period, which was in line with 221 previous finding that the average abundance of this miRNA before birth was eight times higher 
222 than that in postnatal (Qin et al. 2013). miR-486 is directly controlled by SRF, myocardin-related

223 transcription factor-A (MRTF-A), and MyoD, and regulates muscle growth and homeostasis

224 (Small et al. 2010). Moreover, miR-191 could promote cell proliferation and suppress apoptosis

225 in MGC803 cells (Shi et al. 2011). These miRNAs were within the top 10 expressed miRNAs in

226 180-d and 7-y pig muscle, but not in relatively early stages, which indicates that they have

227 regulatory roles in muscle function maintenance after sexual maturity. In contrast, miR-199a is a

228 potential regulator of myogenesis through suppression of WNT-signaling factors and regulation

229 of muscle growth and homeostasis (Alexander et al. 2013); its expression was higher in the E90,

230 0-d, and 30-d stages than in the 180-d and 7-y stages, which indicates that miR-199a is an

231 important myogenic regulatory factor in early fetal and newborn muscle development.

232 Previous study performed by Qin et al. indicated that most of the highly expressed miRNAs in 233 porcine skeletal muscle such as miR-1 and miR-133 will be more functional. Besides the miR-1 234 and miR133a that we found highly expressed in all the stages (McCarthy \& Esser 2007), miR-26a 235 also showed abundant expression (Huang et al. 2008). Taken together, these results indicate that 236 highly abundant miRNAs across various development stages might play important roles in 237 maintenance of vital muscular physiological functions and regulation of skeletal muscle 238 development. Nonetheless, these highly expressed miRNAs also showed dynamic performance 239 across different stages, which indicates typical spatial and temporal-specific expression of 240 miRNAs during muscle development. Undoubtedly, dysregulation of such miRNAs would perturb 241 muscular homeostasis and may even initiate pathogenesis (Eisenberg et al. 2007; McCarthy \& 242 Esser 2007).

\section{Differentially expressed (DE) miRNA during muscle development stages}

244 To determine if miRNA expression significantly differed between libraries, IDEG6 (Romualdi 245 et al. 2003) was employed to normalize calculations between the high-quality sequences in the 246 nine libraries. A unique miRNA was considered differentially expressed when a general chi- 
247 squared test produced P-values $<0.001$ (Li et al. 2011). By applying this criterion, we identified $248505 / 903$ miRNAs (55.92\%) that were differentially expressed during the muscle development 249 stages (Table S6). A previous study demonstrated that miRNAs function in a dose-dependent 250 manner (Carlsbecker et al. 2010); thus, only the relatively more abundant miRNAs ( $>1000$ read 251 counts) were included in the subsequent analysis. Therefore, 167 of 505 (33.07\%) differentially 252 expressed (DE) miRNAs were retained ( $>1000$ read counts).

253 To further characterize variability in miRNA expression profiling, we performed a hierarchical 254 clustering analysis (HCL) based on the DE miRNAs. As shown in Fig. 3, the miRNA expression 255 profiles showed two major clusters: one that included E90, 0-d, and 30-d stages, and one that 256 included 180-d and 7-y stages. This clustering pattern may correspond to the different growth rate 257 of the muscle fibers in different stages (fast-growth stages: E90, 0-d, and 30-d; slow-growth stages: 258 180-d and 7-y) (Dwyer et al. 1993; Rehfeldt et al. 2000). This significant variation in miRNA 259 expression may be responsible, at least in part, for the phenotypic differences (Fig. 1A). 260 Meanwhile, three biological replicates at 180-d and 7-y were highly correlated (180-d: average r $261=0.9532 ; 7-y$ : average $\mathrm{r}=0.9709)$, which indicates high experimental reliability and good 262 reproducibility of replicates (Fig. 3).

263 Furthermore, nine representative DE miRNAs (miR-133a-5p, miR-181a-1-3p, miR-499-5p, 264 miR-320-3p, miR-24-1-3p, miR-214-3p, let-7g-5p, miR-23a-3p, and miR-10b-3p) were chosen

265 for validation by the stem-loop real time (RT)-PCR-based method using three independent 266 samples. The results were consistent with our sequencing result (Pearson $\mathrm{r}=0.875 \pm 0.119, \mathrm{n}=9$;

267 Fig. 4), which indicates that deep sequencing does allow for the successful discovery of miRNAs 268 from pig muscle with high accuracy and efficiency.

\section{Distinct miRNA expression patterns during muscle development}


To visually illustrate the expression pattern of the miRNAs during different development stages, 271 a short time-series expression miner (STEM) analysis was performed for the DE miRNAs. As 272 shown in Fig. 5A, four model profiles had a larger than expected number of genes assigned to the 273 cluster and were significant $(\mathrm{P}<0.05)$. For expression pattern 1, expression level decreased across 274 all five stages. For expression pattern 2, expression level increased from E90 to $0 \mathrm{~d}$, then deceased 275 from $0 \mathrm{~d}$ to $180 \mathrm{~d}$, and remained stable from $180 \mathrm{~d}$ to $7 \mathrm{y}$. For expression pattern 3, expression 276 level decreased from E90 to $180 \mathrm{~d}$, then deceased from $180 \mathrm{~d}$ to $7 \mathrm{y}$. For expression pattern 4 , 277 expression level kept increasing throughout the five stages. The miRNA list and normalized value 278 of expression by STEM software for each pattern was shown in Table S7. These four profiles may 279 indicate the different roles of corresponding miRNAs; therefore, we performed target prediction 280 and Gene Ontology (GO) analysis. As shown in Fig. 5B, the target genes of highly expressed 281 miRNAs in E90 and $0 \mathrm{~d}$ (patterns 1, 2, and 3) were mainly involved in processes related to skeletal 282 muscle development; for example, "cellular component morphogenesis," "embryonic 283 morphogenesis," and "cell morphogenesis involved in differentiation." In mammals, muscle mass 284 is mainly determined by the number and size of muscle fibers (Rehfeldt et al. 2000). The number 285 of muscle fibers is prenatally determined during primary and secondary muscle fiber formation 286 (about 30 to $60 \mathrm{~d}$ post-gestation) (Zhao et al. 2003), whereas postnatal muscle fiber growth is mainly associated with accumulation of muscle-specific proteins (hypertrophy). Therefore, these miRNAs with high expression levels in the embryonic period, which are closely related to cellular morphogenesis and embryonic morphogenesis, may promote differentiation of the muscle fibers in the embryonic period.

In contrast, the target genes of highly expressed miRNAs in the 180-d and 7-y stages (Fig. 5B, 292 pattern 4) were mainly involved in processes such as "apoptosis," "regulation of apoptosis," 293 "regulation of cell death," and "positive regulation of macromolecule biosynthetic process," which 294 are consistent with the results of previous studies (Dirks \& Leeuwenburgh 2005). Postnatal growth 295 of skeletal muscle mainly occurs through increases in muscle fiber length and girth and is inhibited 
296 in relatively older animals (Rehfeldt et al. 2000). Moreover, it has been demonstrated that muscle

297 atrophy induced in middle-aged or older animals is associated with higher amounts of cell

298 apoptosis and death (Dirks \& Leeuwenburgh 2005). This may be the primary reason these miRNAs

299 that play vital roles in apoptosis are highly expressed in middle-aged stage. Particularly, miR-1

300 showed significantly increasing expression level in $180 \mathrm{~d}$ and $7 \mathrm{y}$ stages (Fig. 5A and Table S7). By

301 using both $\mathrm{C} 2 \mathrm{C} 12$ myotubes and dex-induced muscular atrophy mouse models, Kukreti et al.

302 indicated that miR-1 is a muscle-specific microRNA and has a role in promoting muscle atrophy

303 (Kukreti et al. 2013). On the other hand, McCarthy et al. also revealed that miR-1 decreased during

304 mouse skeletal muscle hypertrophy (McCarthy \& Esser 2007). Our result was consistent with the

305 findings in mouse. Hence, miR-1 could be a potential modulator in regulating porcine postnatal

306 skeletal muscle development.

307 These results together indicate that there are obviously disparate roles of miRNAs in different 308 development stages of skeletal muscle, such as enhanced levels of miRNAs related to 309 differentiation and morphogenesis in the embryonic stage. Conversely, there were increasing 310 levels of apoptosis-related miRNAs relatively later in muscle development, especially in the 311 middle-aged stage.

\section{Conclusions}

313 In this study, we performed a comprehensive investigation of miRNA expression pattern in

314 skeletal muscle across various developmental processes, from embryonic to adult stages, in pigs.

315 We identified a number of DE miRNAs that were associated with porcine muscular development.

316 Through function enrichment analysis of DE miRNAs, we found that the highly expressed 317 miRNAs from E90 to $0 \mathrm{~d}$ were mainly involved in macromolecule metabolic, cellular, and 318 embryonic morphogenesis processes, whereas highly expressed miRNAs in the middle-aged stage

319 were mainly involved in apoptosis. Our results indicate that miRNAs act as key regulators of 320 prenatal and postnatal muscle development, and their functions were specific to different stages of 
321 skeletal muscle development. In particular, enhanced levels of apoptosis-related miRNAs in

322 middle-aged pigs revealed possible elevated muscle atrophy during this period. However, pigs

323 with more consecutive ages are needed in further study to uncover the underlying mechanism of

324 refined postnatal pig skeletal muscle development. Our findings will promote further development

325 of pigs as a model organism for human age-related muscle disease research.

326

327

328

329

330

331

332

333

334

335

336

337

338

339

340

341

342

343

344

345

346

347

348

349

350

351

352

353

354

355

\section{References}

Alexander M, Kawahara G, Motohashi N, Casar J, Eisenberg I, Myers J, Gasperini M, Estrella E, Kho A, and Mitsuhashi S. 2013. MicroRNA-199a is induced in dystrophic muscle and affects WNT signaling, cell proliferation, and myogenic differentiation. Cell Death \& Differentiation 20:1194-1208.

Bartel DP. 2009. MicroRNAs: target recognition and regulatory functions. cell 136:215-233.

Berezikov E. 2011. Evolution of microRNA diversity and regulation in animals. Nature Reviews Genetics 12:846-860.

Bhathena SJ, Berlin E, and Johnson WA. 1996. The Minipig as a Model for the Study of Aging in Humans. Advances in Swine in Biomedical Research: Springer, 571-579.

Brooks SV, and Faulkner JA. 1988. Contractile properties of skeletal muscles from young, adult and aged mice. The Journal of physiology 404:71.

Carlsbecker A, Lee J-Y, Roberts CJ, Dettmer J, Lehesranta S, Zhou J, Lindgren O, MorenoRisueno MA, Vatén A, and Thitamadee S. 2010. Cell signalling by microRNA165/6 directs gene dose-dependent root cell fate. Nature 465:316-321.

Chen C, Deng B, Qiao M, Zheng R, Chai J, Ding Y, Peng J, and Jiang S. 2012. Solexa sequencing identification of conserved and novel microRNAs in backfat of Large White and Chinese Meishan pigs. PloS one 7:e31426.

Crist CG, Montarras D, Pallafacchina G, Rocancourt D, Cumano A, Conway SJ, and Buckingham M. 2009. Muscle stem cell behavior is modified by microRNA-27 regulation of Pax3 expression. Proceedings of the National Academy of Sciences 106:13383-13387.

Daw CK, Starnes JW, and White TP. 1988. Muscle atrophy and hypoplasia with aging: impact of training and food restriction. Journal of Applied Physiology 64:2428-2432.

Di Giovanni S, Molon A, Broccolini A, Melcon G, Mirabella M, Hoffman EP, and Servidei S. 2004. Constitutive activation of MAPK cascade in acute quadriplegic myopathy. Annals of neurology 55:195-206.

Dirks A, and Leeuwenburgh C. 2002. Apoptosis in skeletal muscle with aging. American Journal of Physiology-Regulatory, Integrative and Comparative Physiology 282:R519-R527.

Dirks AJ, and Leeuwenburgh C. 2005. The role of apoptosis in age-related skeletal muscle atrophy. Sports Medicine 35:473-483. 
356

357

358

359

360

361

362

363

364

365

366

367

368

369

370

371

372

373

374

375

376

377

378

379

380

381

382

383

384

385

386

387

388

389

390

391

392

393

394

Dogra C, Changotra H, Wedhas N, Qin X, Wergedal JE, and Kumar A. 2007. TNF-related weak inducer of apoptosis (TWEAK) is a potent skeletal muscle-wasting cytokine. The FASEB Journal 21:1857-1869.

Drummond MJ, McCarthy JJ, Sinha M, Spratt HM, Volpi E, Esser KA, and Rasmussen BB. 2011. Aging and microRNA expression in human skeletal muscle: a microarray and bioinformatics analysis. Physiological genomics 43:595-603.

Dwyer C, Fletcher J, and Stickland N. 1993. Muscle cellularity and postnatal growth in the pig. Journal of Animal Science 71:3339-3343.

Eisenberg I, Eran A, Nishino I, Moggio M, Lamperti C, Amato AA, Lidov HG, Kang PB, North KN, and Mitrani-Rosenbaum S. 2007. Distinctive patterns of microRNA expression in primary muscular disorders. Proceedings of the National Academy of Sciences 104:1701617021.

Ernst J, and Bar-Joseph Z. 2006. STEM: a tool for the analysis of short time series gene expression data. BMC bioinformatics 7:191.

Evans WJ, and Lexell J. 1995. Human aging, muscle mass, and fiber type composition. The Journals of Gerontology Series A: Biological Sciences and Medical Sciences 50:11-16.

Fiedler I, Rehfeldt C, Dietl G, and Ender K. 1997. Phenotypic and genetic parameters of muscle fiber number and size. J Anim Sci 75:165.

Güller I, and Russell AP. 2010. MicroRNAs in skeletal muscle: their role and regulation in development, disease and function. The Journal of physiology 588:4075-4087.

Gambardella S, Rinaldi F, Lepore SM, Viola A, Loro E, Angelini C, Vergani L, Novelli G, and Botta A. 2010. Research Overexpression of microRNA-206 in the skeletal muscle from myotonic dystrophy type 1 patients.

Hamrick MW, Herberg S, Arounleut P, He H-Z, Shiver A, Qi R-Q, Zhou L, Isales CM, and Mi QS. 2010. The adipokine leptin increases skeletal muscle mass and significantly alters skeletal muscle miRNA expression profile in aged mice. Biochemical and biophysical research communications 400:379-383.

Hooper A. 1981. Length, diameter and number of ageing skeletal muscle fibres. Gerontology 27:121-126.

Hou X, Tang Z, Liu H, Wang N, Ju H, and Li K. 2012. Discovery of MicroRNAs associated with myogenesis by deep sequencing of serial developmental skeletal muscles in pigs.

Howe E, Holton K, Nair S, Schlauch D, Sinha R, and Quackenbush J. 2010. Mev: multiexperiment viewer. Biomedical Informatics for Cancer Research: Springer, 267-277.

Huang DW, Sherman BT, and Lempicki RA. 2008a. Systematic and integrative analysis of large gene lists using DAVID bioinformatics resources. Nature protocols 4:44-57.

Huang T-H, Zhu M-J, Li X-Y, and Zhao S-H. 2008b. Discovery of porcine microRNAs and profiling from skeletal muscle tissues during development. PloS one 3:e3225.

Huang Z, Chen X, Yu B, He J, and Chen D. 2012. MicroRNA-27a promotes myoblast proliferation by targeting myostatin. Biochem Biophys Res Commun 423:265-269. 
395

396

397

398

399

400

401

402

403

404

405

406

407

408

409

410

411

412

413

414

415

416

417

418

419

420

421

422

423

424

425

426

427

428

429

430

431

432

433

10.1016/j.bbrc.2012.05.106

Jackman RW, and Kandarian SC. 2004. The molecular basis of skeletal muscle atrophy. American Journal of Physiology-Cell Physiology 287:C834-C843.

Jagoe RT, and Goldberg AL. 2001. What do we really know about the ubiquitin-proteasome pathway in muscle atrophy? Current Opinion in Clinical Nutrition \& Metabolic Care 4:183-190.

Janssen I, Heymsfield SB, Wang Z, and Ross R. 2000. Skeletal muscle mass and distribution in 468 men and women aged 18-88 yr. Journal of Applied Physiology 89:81-88.

Jemal A, Siegel R, Ward E, Hao Y, Xu J, Murray T, and Thun MJ. 2008. Cancer statistics, 2008. CA: a cancer journal for clinicians 58:71-96.

Krek A, Grün D, Poy MN, Wolf R, Rosenberg L, Epstein EJ, MacMenamin P, da Piedade I, Gunsalus KC, and Stoffel M. 2005. Combinatorial microRNA target predictions. Nature genetics 37:495-500.

Kukreti H, Amuthavalli K, Harikumar A, Sathiyamoorthy S, Feng PZ, Anantharaj R, Tan SL, Lokireddy S, Bonala S, Sriram S, McFarlane C, Kambadur R, and Sharma M. 2013. Muscle-specific microRNA1 (miR1) targets heat shock protein 70 (HSP70) during dexamethasone-mediated atrophy. $J$ Biol Chem 288:6663-6678. 10.1074/jbc.M112.390369

Lewis BP, Burge CB, and Bartel DP. 2005. Conserved seed pairing, often flanked by adenosines, indicates that thousands of human genes are microRNA targets. cell 120:15-20.

Li HY, Xi QY, Xiong YY, Liu XL, Cheng X, Shu G, Wang SB, Wang LN, Gao P, and Zhu XT. 2012. Identification and comparison of microRNAs from skeletal muscle and adipose tissues from two porcine breeds. Animal genetics 43:704-713.

Li M, Liu Y, Wang T, Guan J, Luo Z, Chen H, Wang X, Chen L, Ma J, and Mu Z. 2011. Repertoire of porcine microRNAs in adult ovary and testis by deep sequencing. International journal of biological sciences 7:1045.

Lunney JK. 2007. Advances in swine biomedical model genomics. International journal of biological sciences 3:179.

Lynch GS. 2001. Therapies for improving muscle function in neuromuscular disorders. Exercise and sport sciences reviews 29:141-148.

McCarthy JJ, and Esser KA. 2007. MicroRNA-1 and microRNA-133a expression are decreased during skeletal muscle hypertrophy. Journal of Applied Physiology 102:306-313.

Sharma M, Juvvuna PK, Kukreti H, and McFarlane C. 2014. Mega roles of microRNAs in regulation of skeletal muscle health and disease. Front Physiol 5:239. 10.3389/fphys.2014.00239

Nair KS. 2005. Aging muscle. The American journal of clinical nutrition 81:953-963.

Navarro A, Lopez-Cepero J, and Sánchez dPM. 2001. Skeletal muscle and aging. Frontiers in bioscience: a journal and virtual library 6:D26-44.

Nelson P, Kiriakidou M, Sharma A, Maniataki E, and Mourelatos Z. 2003. The microRNA world: 
434

435

436

437

438

439

440

441

442

443

444

445

446

447

448

449

450

451

452

453

454

455

456

457

458

459

460

461

462

463

464

465

466

467

468

469

470 small is mighty. Trends in biochemical sciences 28:534-540.

Pollack M, Phaneuf S, Dirks A, and Leeuwenburgh C. 2002. The role of apoptosis in the normal aging brain, skeletal muscle, and heart. Annals of the New York Academy of Sciences 959:93-107.

Qin L, Chen Y, Liu X, Ye S, Yu K, Huang Z, Yu J, Zhou X, Chen H, and Mo D. 2013. Integrative analysis of porcine microRNAome during skeletal muscle development. PLoS One 8:e72418. 10.1371/journal.pone.0072418

Rehfeldt C, Fiedler I, Dietl G, and Ender K. 2000. Myogenesis and postnatal skeletal muscle cell growth as influenced by selection. Livestock Production Science 66:177-188.

Romualdi C, Bortoluzzi S, d'Alessi F, and Danieli GA. 2003. IDEG6: a web tool for detection of differentially expressed genes in multiple tag sampling experiments. Physiological genomics 12:159-162.

Sartorelli V, and Fulco M. 2004. Molecular and cellular determinants of skeletal muscle atrophy and hypertrophy. Science Signaling 2004:re11-re11.

Shi X, Su S, Long J, Mei B, and Chen Y. 2011. MicroRNA-191 targets N-deacetylase/Nsulfotransferase 1 and promotes cell growth in human gastric carcinoma cell line MGC803. Acta biochimica et biophysica Sinica 43:849-856.

Small EM, O’Rourke JR, Moresi V, Sutherland LB, McAnally J, Gerard RD, Richardson JA, and Olson EN. 2010. Regulation of PI3-kinase/Akt signaling by muscle-enriched microRNA486. Proceedings of the National Academy of Sciences 107:4218-4223.

Tang Y, Zheng J, Sun Y, Wu Z, Liu Z, and Huang G. 2009. MicroRNA-1 regulates cardiomyocyte apoptosis by targeting Bcl-2. International heart journal 50:377-387.

Wong CF, and Tellam RL. 2008. MicroRNA-26a targets the histone methyltransferase Enhancer of Zeste homolog 2 during myogenesis. Journal of Biological Chemistry 283:9836-9843.

Xie S-S, Li X-Y, Liu T, Cao J-H, Zhong Q, and Zhao S-H. 2011. Discovery of porcine microRNAs in multiple tissues by a Solexa deep sequencing approach. PloS one 6:e16235.

Zhao S-H, Nettleton D, Liu W, Fitzsimmons C, Ernst C, Raney N, and Tuggle C. 2003. Complementary DNA macroarray analyses of differential gene expression in porcine fetal and postnatal muscle. Journal of Animal Science 81:2179-2188.

Zhou Y, Tang X, Song Q, Ji Y, Wang H, Jiao H, Ouyang H, and Pang D. 2013. Identification and characterization of pig embryo microRNAs by Solexa sequencing. Reproduction in domestic animals 48:112-120. 
Figures and legends
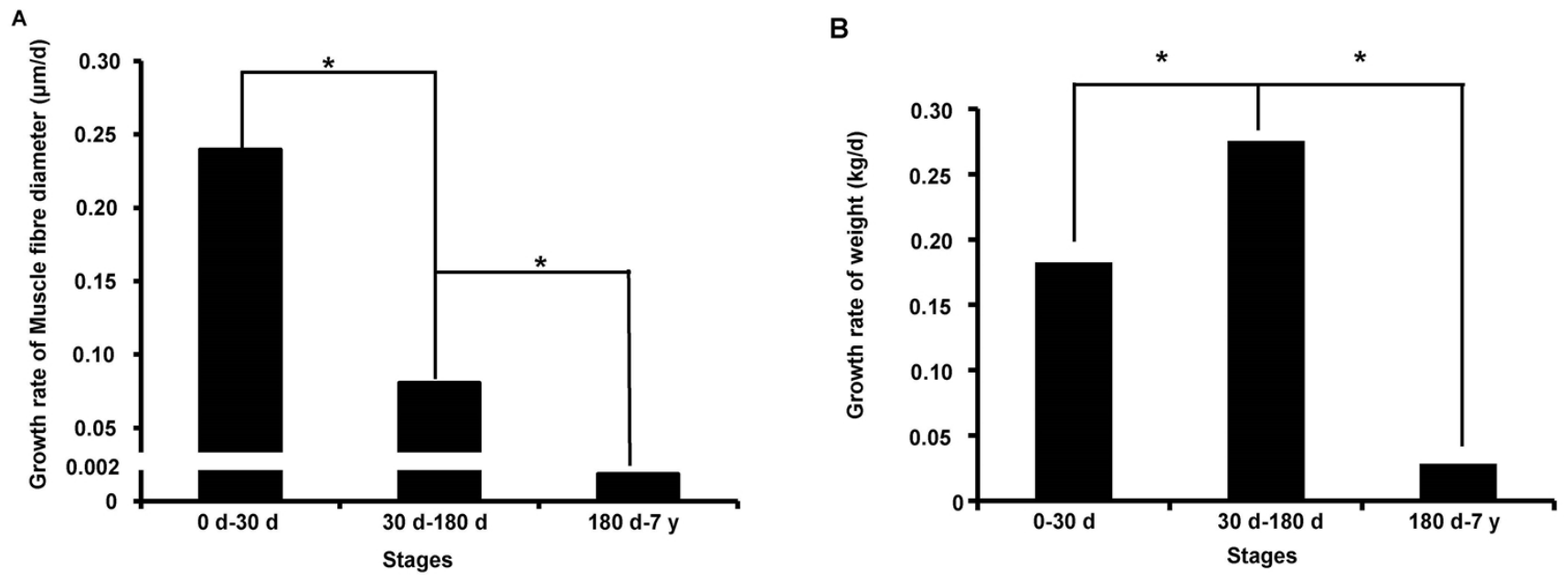

Fig. 1 Growth rate of muscle fiber diameter (A) and weight (B) during the postnatal muscle development stage. Three replicates were used when performed the statistical analysis between different development stages. "**" indicates significant difference (Student's $t$-test, $p<0.05$ ).

485

486

487

488

489 
490

491

492

493

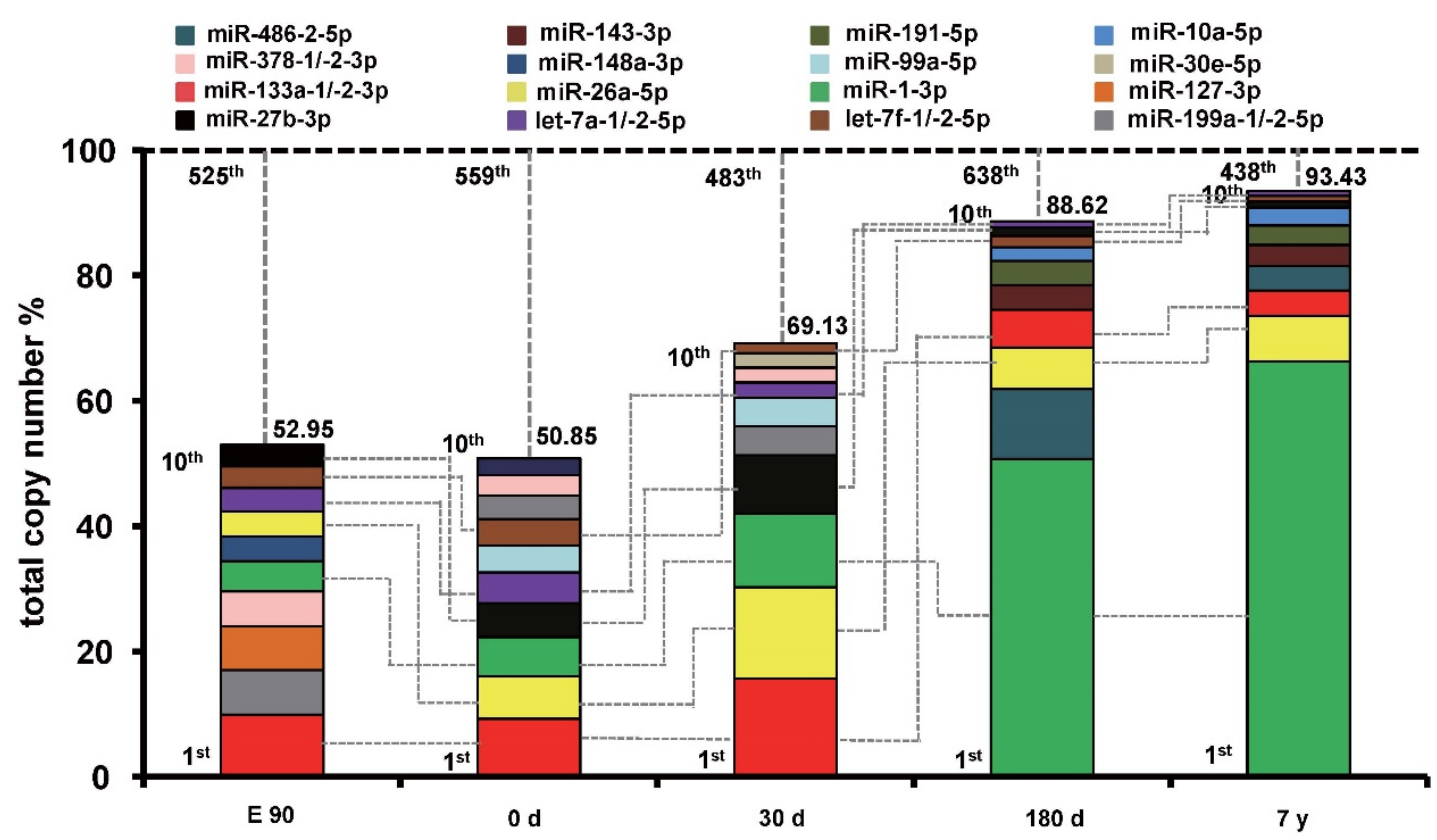

495

496

497

498

499

500

501

502

503

504

505

506

507

Fig. 2 Top 10 unique miRNAs with the highest expression levels during the five muscle development stages. Plot of the unique miRNAs versus their total copy number $\%$ of all unique miRNAs for each library. The dashed vertical lines represent the cumulative $\%$ of the top 10 unique miRNAs in total counts of all unique miRNAs. The 6 miRNAs that are present in the top 10 miRNAs in all libraries are connected by lines. 


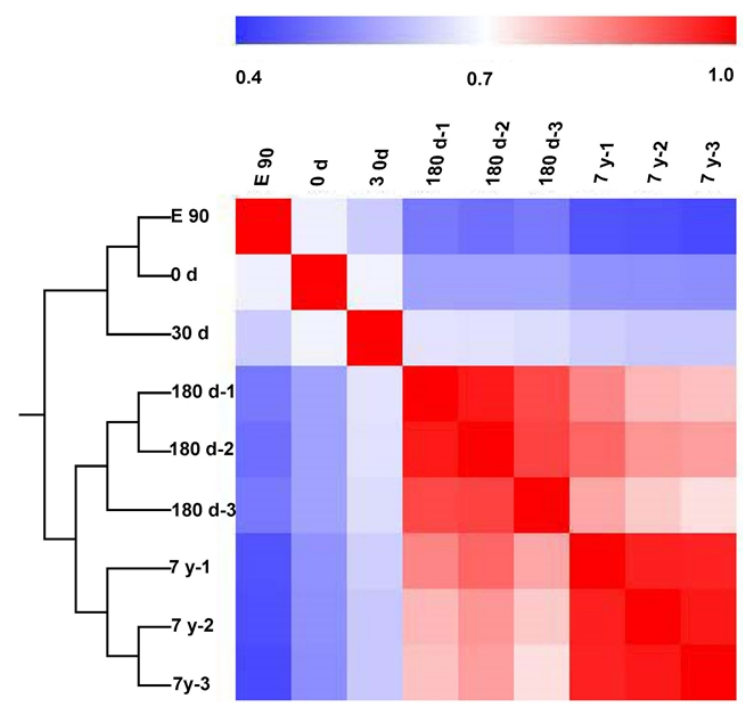
correlations of the counts of 167 differentially expressed miRNAs between nine miRNA libraries.

513

514

515

516

517

518

519

520

521 


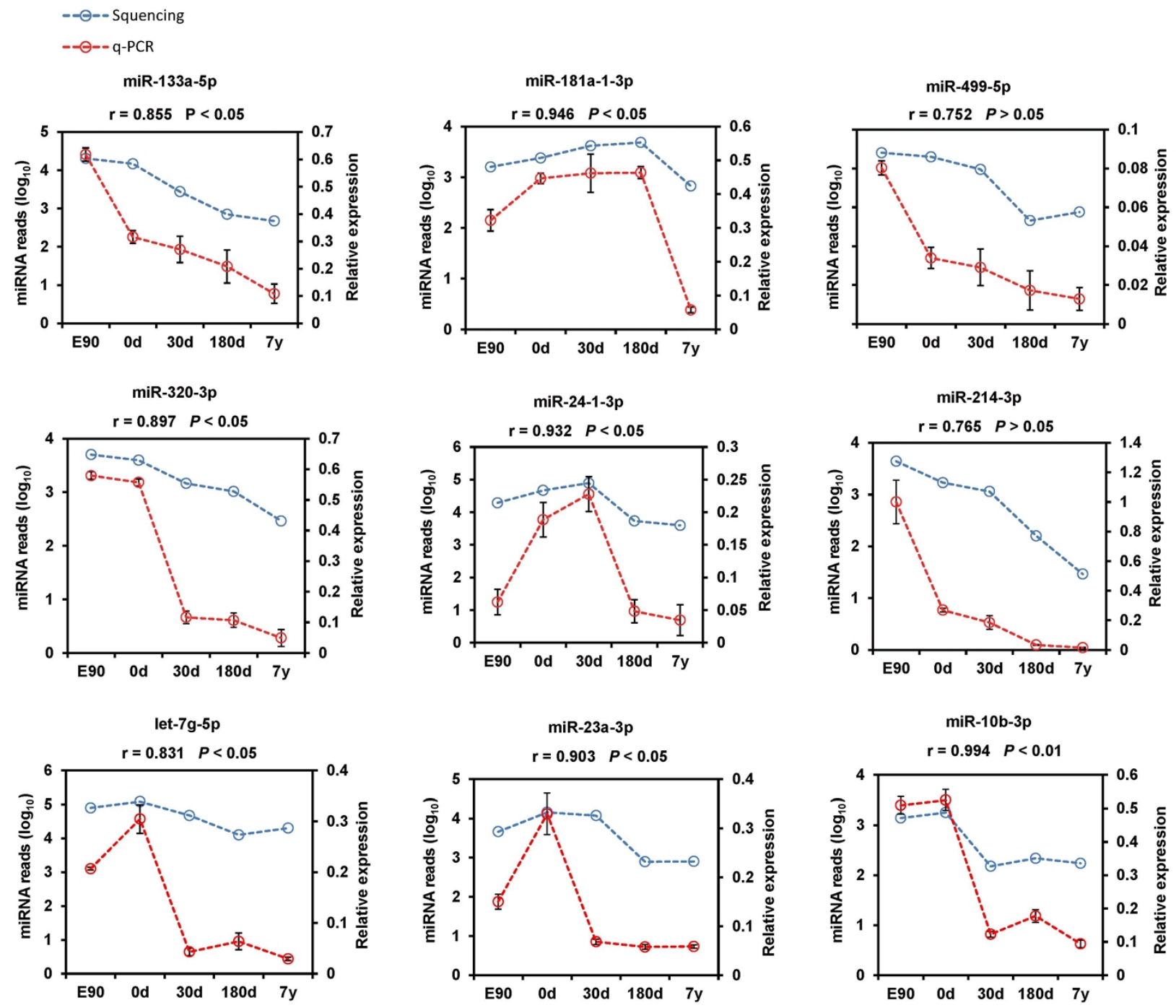
representative DE miRNAs. The SPSS software was used to calculate the Pearson correlation coefficient (r) and corresponding significance value $(P)$. 
A
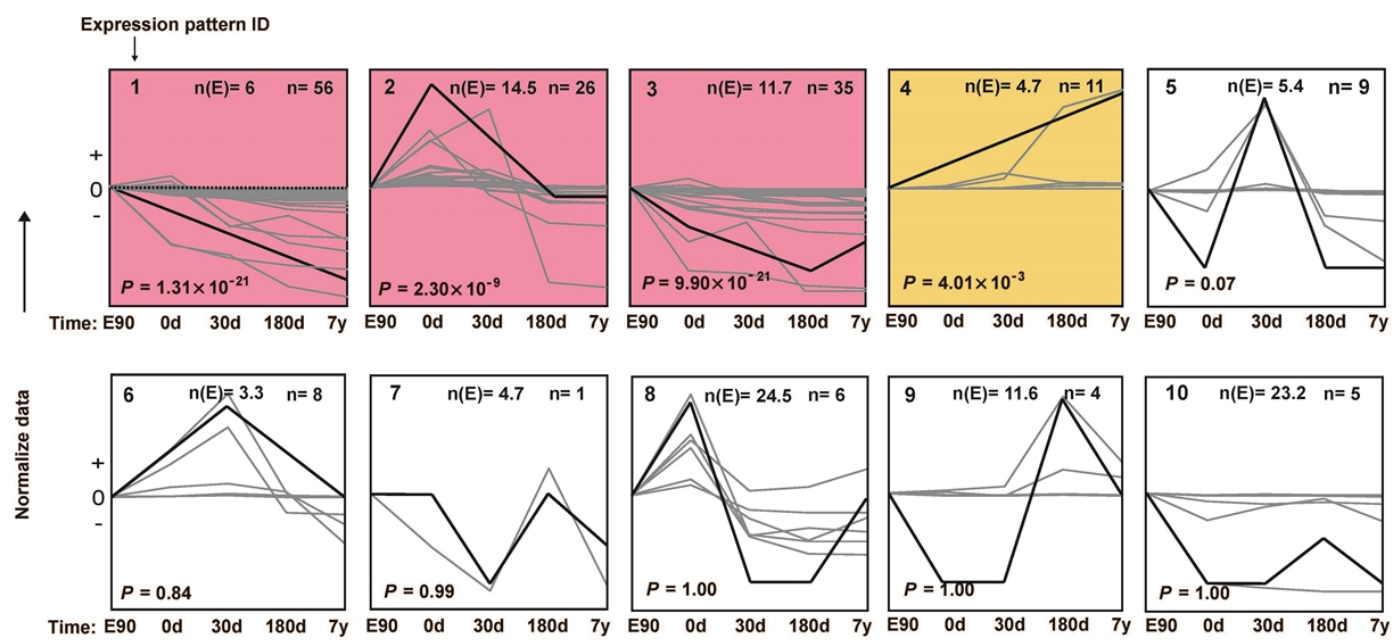

B

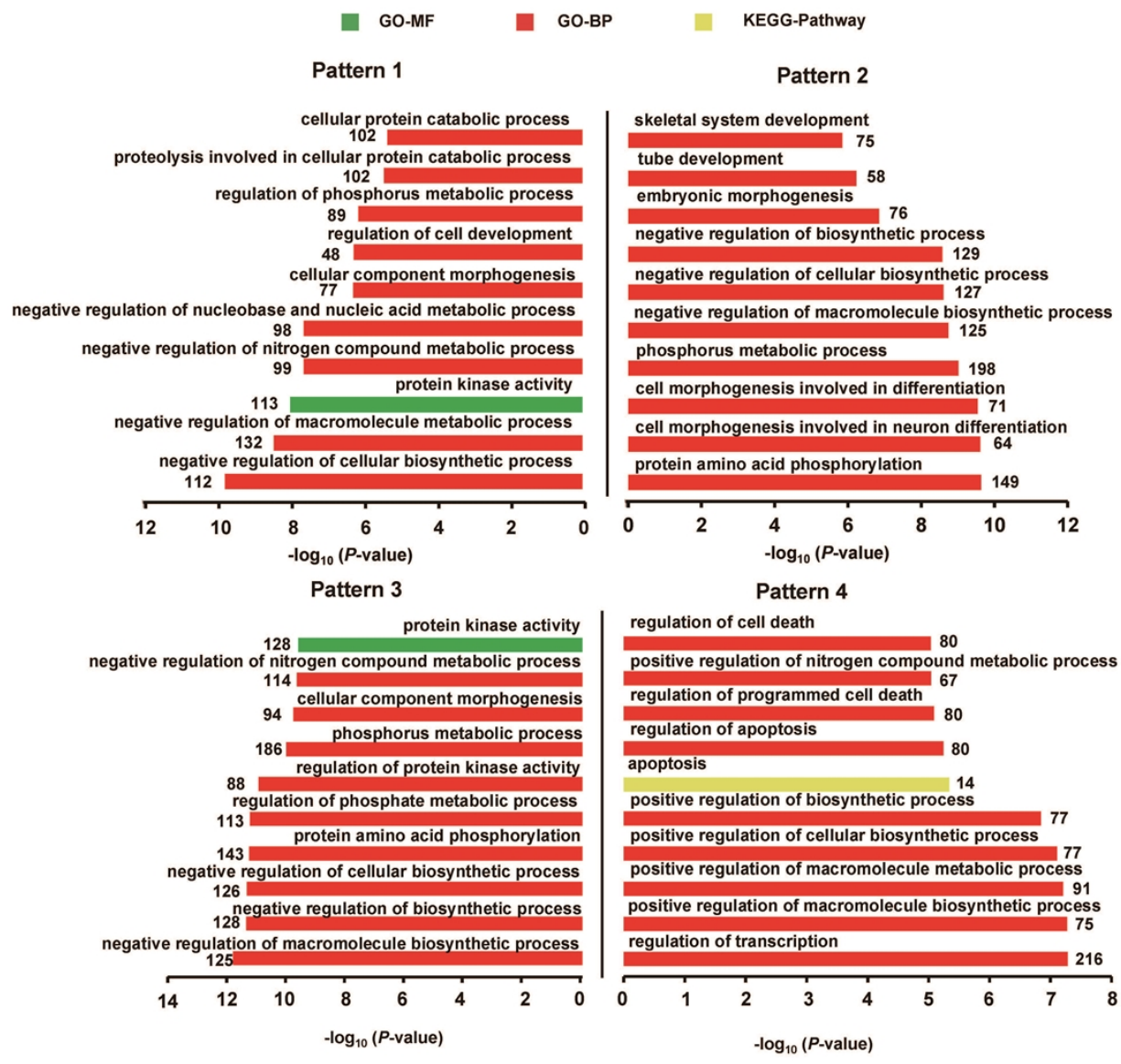

Fig. 5 Expression patterns for differentially expressed miRNAs. (A) The trends of 167 differentially expressed miRNAs were generally divided into 10 clusters based on their dynamic expression patterns among the five muscle development stages. 
532

533

534

535

536

537

538

539

540

541

542

543

544

545

546

547

548

549

550

551

552

553

554

555

556

557

558

Numbers in the top left corner indicate the cluster number. Numbers in the top right corner indicate the number of miRNAs in each cluster. The colored clusters indicate clusters that were significantly enriched by miRNAs $(P<0.05)$ (Fisher's exact test). (B) Kyoto Encyclopedia of Genes and Genomes (KEGG) pathways and Gene Ontology-Biological Processes (GO-BP) enriched for target genes of the four significant miRNA clusters. The $P$ values were calculated using Benjamini-corrected modified Fisher's exact test. 
559

560

561

562

\section{Tables}

564 Table 1 Porcine and conserved miRNAs detected in nine sRNA libraries

565

\begin{tabular}{cccccccccc}
$\begin{array}{c}\text { Group (number } \\
\text { of pre- }\end{array}$ & $\mathrm{E} 90$ & $\mathrm{Od}$ & $30 \mathrm{~d}$ & $180 \mathrm{~d}-1$ & $180 \mathrm{~d}-2$ & $180 \mathrm{~d}-3$ & $7 \mathrm{y}-1$ & $7 \mathrm{y}-2$ & $7 \mathrm{y}-3$ \\
\hline $\begin{array}{c}\text { Porcine known } \\
\text { miRNAs } \\
\text { Porcine } \\
\text { conserved } \\
\ldots\end{array}$ & $229 / 379$ & $221 / 372$ & $213 / 340$ & $212 / 321$ & $205 / 312$ & $205 / 322$ & $186 / 260$ & $175 / 233$ & $186 / 272$ \\
$\begin{array}{c}\text { Porcine putative } \\
\text { new miRNAs }\end{array}$ & $84 / 83$ & $55 / 78$ & $47 / 67$ & $47 / 62$ & $41 / 55$ & $37 / 49$ & $25 / 28$ & $24 / 28$ & $31 / 37$ \\
\hline
\end{tabular}

566

567

568

569

570

571

572

573

574

575

576

577

578

579 


\section{Supplemental Information}

587 Fig. S1 Muscle fiber diameter and body weight during the postnatal muscle development 588 stage.

589 Fig. S2 Distribution of raw reads in each library after applying a series of filters.

590 Fig. S3 Length distribution of mappable reads.

591 Fig. S4 Cumulative distribution of unique miRNAs.

592 Table S1 Primer sequences used for q-PCR experiments.

593 Table S2 Profile of known porcine miRNAs (based on miRBase 19.0) with genome locations.

594 Table S3 Profile of other mammalian miRNAs (based on miRBase 19.0).

595 Table S4 Profile of porcine candidate miRNAs.

596 Table S5 Unique porcine miRNAs identified in this study.

597 Table S6 DE miRNAs during muscle development stages.

598 Table S7 Significant miRNA expression patterns during porcine muscle development.

599

600 